\title{
The Study of Nonlinear Flutter Analysis Method for an Aircraft Full Composite Wing with High-aspect-ratio

\author{
Sheng-Jun $\mathrm{QIAO}^{\mathrm{a}}$, Hang-Shan GAO ${ }^{\text {b, }}$, Jun-Ran ZHANG
}

\author{
Department of Engineering Mechanics, Northwestern Polytechnical University, Advanced Material \\ Testing Center, Xi'an 710129, China \\ aqiaoshengjun123@163.com, bgaohs@nwpu.edu.cn \\ ${ }^{*}$ Corresponding author
}

Keywords: Nonlinear Flutter, Corotational, Thin Shell Element, High-aspect-ratio Wing.

\begin{abstract}
A nonlinear flutter analysis method using CFD/CSD was presented in this paper. The computational method couples a nonlinear finite element method model with CFD solver. The structural analysis was based on a 4-node thin shell element and a new corotational (CR) formulation, which can be employed to analyze geometric nonlinearities arising from large deflections. The expression of tangent stiffness equations under geometric nonlinear structure was derived. Aerodynamic load was calculated by CFD solver with the Navier-Stokes equations as the control equations. This paper showed that the geometric nonlinear method is computationally high efficient and good agreement by examined. The geometric nonlinear method can be successfully applied for the nonlinear flutter analysis to full composite wing with high-aspect-ratio. The flutter speed of the composite wing is near $0.65 \mathrm{Ma}$.
\end{abstract}

\section{Introduction}

Nonlinear flutter for composite wing contains two sections with structure nonlinear and aerodynamic nonlinear. Based on nonlinear finite element method and CFD computational methodology, CFD/CSD coupling analyses is play an important role in solving nonlinear flutter for composite wing.

Based on a high-fidelity nonlinear structural model and a linear vortex lattice aerodynamic model, flutter and limit-cycle oscillations of a delta-wing model were studied ${ }^{[1]}$. Aeroelastic model consisted of Vortex-lattice aerodynamics coupled with von Karman plate model for delta wing ${ }^{[2]}$. Computational model includes a well-validated Euler finite difference solver coupled to a high-fidelity finite element structural solver, flutter and limit cycle oscillation behavior of a cropped delta wing were investigated ${ }^{[3]}$. Based on nonlinear finite element, an aeroelastic coupling procedure was performed with loosing coupling CFD/CSD at the same physics time step $^{[4]}$. A numerical method coupling $\mathrm{N}-\mathrm{S}$ equations and structural modal equations for predicting three-dimensional transonic wing flutter was described, and modal approach was used for predicting structural response ${ }^{[5]}$.

Based on CR description, a CR description has already been applied in aeroelasticity ${ }^{[6]}$. A two-dimensional formulation based on CR description was developed by Crisfield ${ }^{[7]}$. Based on CR theory, the expressions of tangent stiffness equations and internal forces of 3D shell element under geometric nonlinear structure were derived to analysis nonlinear aeroelasticity ${ }^{[8]}$. Quadratic and cubic aerodynamic nonlinearities as well as cubic geometrical nonlinearities were considered in nonlinear aeroelastic behavior ${ }^{[9]}$.

Geometric nonlinearities influences flutter characteristics of a high-aspect-ratio wing greatly. The objective of the paper is to present a nonlinear flutter analysis method, which based on a high efficiency geometric nonlinearities analysis method.

\section{Nonlinear Method}

Most of the finite models were established by thin shell elements for thin wall structures in 
project. Such as the structures CR formulation was presented as a more convenient analysis, which was confirmed by many numerical illustrations and tests. The motion of the element from the initial to the final deformed configuration can be splited into two stages. The first step is a rigid translation and rotation of the undeformed element. The second step is a local deformation displacement and rotation with respect to the local frame.

\section{Transformation of the Rigid Rotations}

The transformation of the rigid rotations from the initial local coordinate system to the final deformed configuration is achieved by the orthogonal rotation matrix $R_{r}$ Pacoste $^{[10]}$, defined in Fig.1.

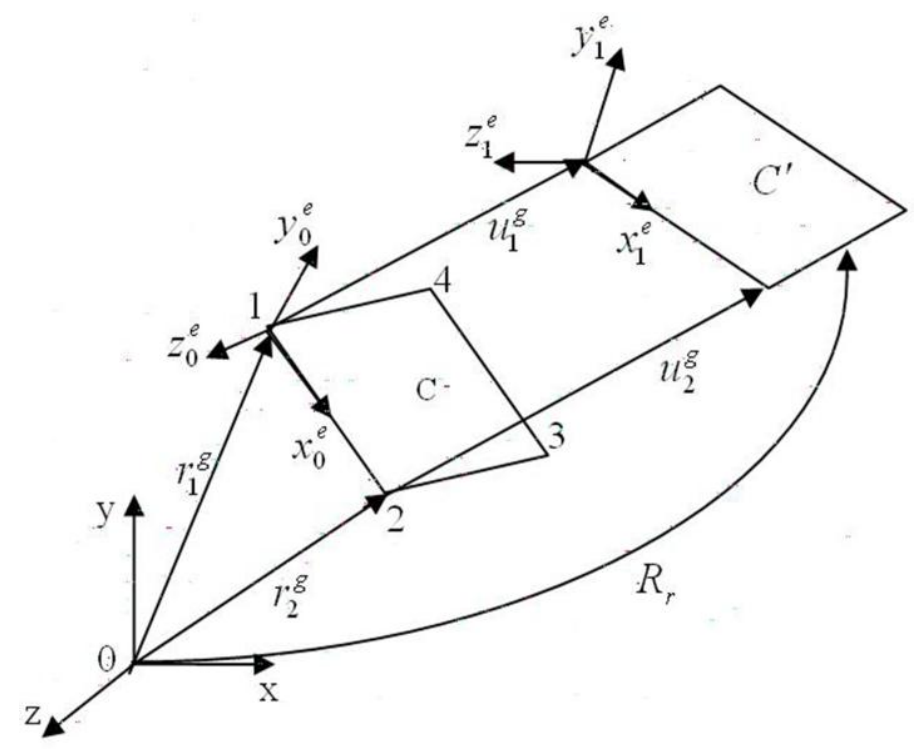

Fig.1 Element Kinematics and Coordinate Systems

The rotation is relative to local axes, so $R_{r}$ can be written as

$$
R_{r}=\left[\begin{array}{lll}
e_{1} & e_{2} & e_{3}
\end{array}\right]
$$

with

$$
\begin{aligned}
& e_{1}=\frac{\left(r_{j}^{g}+u_{j}^{g}-r_{i}^{g}-u_{i}^{g}\right)}{\left\|r_{j}^{g}+u_{j}^{g}-r_{i}^{g}-u_{i}^{g}\right\|} \\
& e_{3}=\frac{x_{j} \times x_{k}}{\left\|x_{j} \times x_{k}\right\|} \\
& e_{2}=e_{1} \times e_{3}
\end{aligned}
$$

where $e_{i}(i=1,2,3)$ denote the orthonormal basis vectors of the local frame in the current deformed configuration.

\section{Transformation Matrix}

The transformation matrix is needed to convert the internal force and tangent stiffness matrix from local to global coordination. In the global coordinate, the 4-node element displacement and force vector is defined as

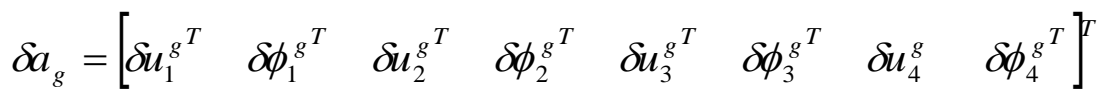


The element global internal force can be defined as

$$
F=\left[\begin{array}{llllllll}
F_{1}^{T} & M_{1}^{T} & F_{2}^{T} & M_{2}^{T} & F_{3}^{T} & M_{3}^{T} & F_{4}^{T} & M_{4}^{T}
\end{array}\right]=T^{T} f_{l}
$$

with

$$
\begin{aligned}
& T=P E^{T} B_{m} \\
& P_{i j}=\left[\begin{array}{ll}
\frac{\partial u_{i}^{-e}}{\partial u_{j}^{e}} & \frac{\partial u_{i}^{-e}}{\partial \theta_{j}^{e}} \\
\frac{\partial \theta_{i}^{e}}{\partial u_{j}^{e}} & \frac{\partial \theta_{i}^{e}}{\partial \theta_{j}^{e}}
\end{array}\right]=I \delta_{\mathrm{ij}}-A_{i} G_{j}^{T} \\
& G_{j}=\left[\begin{array}{lll}
G_{1} & G_{2} & G_{3}
\end{array}\right] \\
& E=\operatorname{diag}\left(R_{r}, R_{r}, R_{r}, R_{r}, R_{r}, R_{r}, R_{r}, R_{r}\right) \\
& B_{m}=\operatorname{diag}\left(I_{3}, T_{m}\left(\phi_{1}\right), I_{3}, T_{m}\left(\phi_{2}\right), I_{3}, T_{m}\left(\phi_{3}\right), I_{3}, T_{m}\left(\phi_{4}\right)\right)
\end{aligned}
$$

where $u_{i}^{g}(i=1,2,3,4)$ are the global displacement vectors of the nodes, $\delta \phi_{i}^{g}(i=1,2,3,4)$ denotes the spatial angular variations. $T_{g}$ is the transformation matrix with $20 \times 20$ blocks from local to global coordinate, $P$ is the matrix which extracts the deformational part from the total displacements. More detail process of the element kinematics is defined by local and global displacement and rotation vector in ${ }^{[10,11]}$.

\section{Element Internal Force and Tangent Stiffness Matrix}

The established transformation matrix by co-rotational can be found in this paper, so the element global tangent stiffness matrix is defined as

$$
K_{T}^{e}=T^{T} K_{l}^{e} T+K_{\sigma}
$$

with

$$
\begin{aligned}
& K_{\sigma}=\operatorname{diag}\left(0_{3}, K_{\sigma 1}, 0_{3}, K_{\sigma 2,} 0_{3}, K_{\sigma 3}, 0_{3}, K_{\sigma 4}\right) \\
& K_{\sigma i}=\partial T^{T}\left(M_{g i}\right) / \partial \phi_{i}, \quad i=1,2,3,4
\end{aligned}
$$

In Eq. (6), the element tangent stiffness matrix is reduced from a $24 \times 24$ matrix to a $20 \times 20$ matrix. A bilinear in-plane displacement field is introduced for the coupling of in-plane and bending actions, the local stiffness matrix avoiding shear locking is calculated by $3 \times 3$ Gauss integral, which is more efficient to save computational times.

\section{Nonlinear Method Validation}

The purpose of the example is to verify the effect of the present element in double-curved thin shell structure, which is always used in many engineering structures and shows significant different nonlinear. $32 \times 20$ meshes and lay-ups of $[45 / 90 / 0 /-45]_{S}$ are used in this analysis. The length of $b=2000 \mathrm{~mm}$ and width of $\mathrm{a}=1250 \mathrm{~mm}$ are shown in Fig.2, and the total thickness of the laminates is $h=3.2 \mathrm{~mm}$. Geometry and material properties are used as

$$
\begin{aligned}
& L=320 \mathrm{~mm}, \quad h=2.4 \mathrm{~mm}, \quad E_{1}=1.334 \times 10^{5} \mathrm{~N} / \mathrm{mm}, \quad E_{2}=1.008 \times 10^{4} \mathrm{~N} / \mathrm{mm} \\
& G_{12}=G_{13}=G_{23}=4.9 \times 10^{3} \mathrm{~N} / \mathrm{mm}, \quad v_{12}=0.31
\end{aligned}
$$




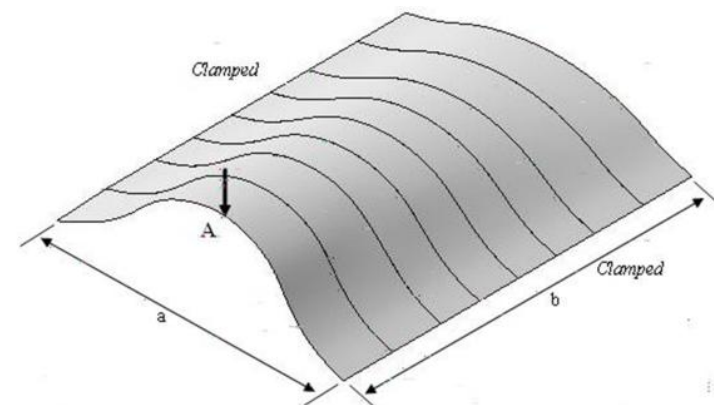

Fig.2 Geometry Proprieties of Double-curved thin Shell Structure

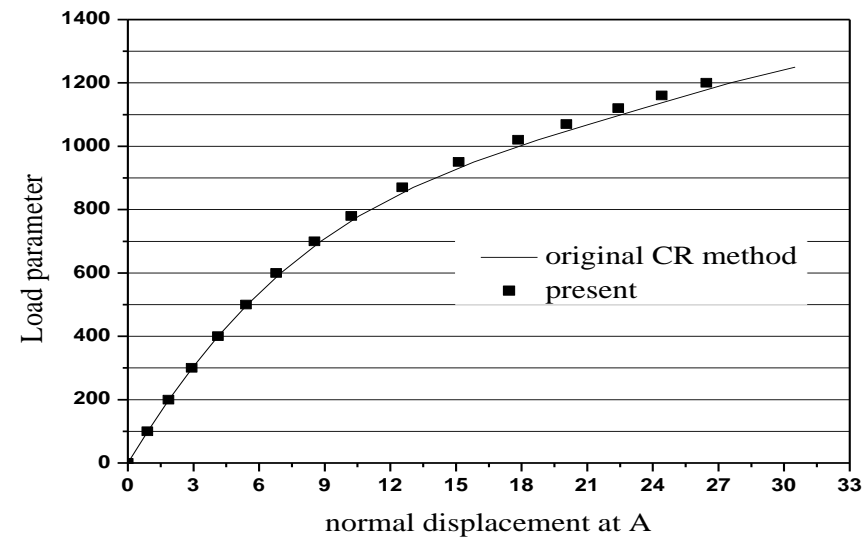

Fig.3 Load-displacement Curve of Double Thin Shell Structure

The normal displacement at A of the double-curved thin shell structure is compared with the original CR method in Fig.3, the CPU time of the original and present are 258s and 167s, respectively. The results showed that the present element is acceptable for the double-curved structure. The 4-node thin shell element is $35.3 \%$ faster than the original element.

\section{Nonlinear Flutter Analysis}

The nonlinear analysis method with established in section 2 is applied to flutter analysis for full composite wing with high-aspect-ratio. Aerodynamic load is calculated by CFD solver with the Navier-Stokes equations as the control equations. Based on lagrange's equations, the flutter kinetic equation of multiple degree airplane is defined as

$$
M \ddot{q}+C \dot{q}+K q=Q
$$

Where $M, C, K, q$ and $Q$ are generalized mass, generalized damper, generalized stiffness, generalized displacement and generalized load matrices, respectively. The Eq. (8) can be transferred into first order differential equation set, which is written as

$$
[A]\{\dot{X}\}+[B]\{X\}=[F]
$$

with

$$
[A]=\left[\begin{array}{cc}
C & M \\
1 & 0
\end{array}\right],[B]=\left[\begin{array}{cc}
K & 0 \\
0 & -1
\end{array}\right],\{\dot{X}\}=\left\{\begin{array}{l}
\dot{q} \\
\ddot{q}
\end{array}\right\},\{X\}=\left\{\begin{array}{l}
q \\
\dot{q}
\end{array}\right\},\{F\}=\left\{\begin{array}{l}
Q \\
0
\end{array}\right\}
$$

Runge-Kutta method is adopted to resolve the Eq. (9), the time histories of the three generalized displacements are gained. 
A finite element model of the high-aspect-ratio wing is shown in Figure 4. The whole computation zone and wing surface grids for aerodynamic analysis are shown in Figure 5.

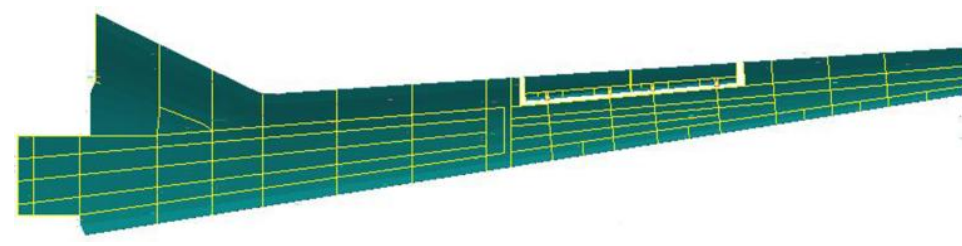

Fig.4 Wing Finite Element Model

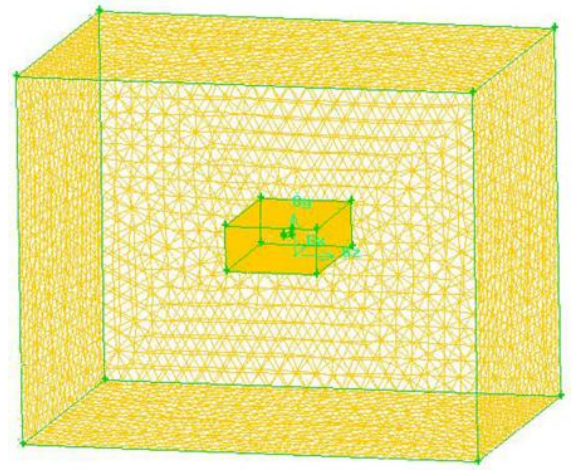

Fig.5 The Whole Computation Zone

Here, two cases with different flight speeds are studied, in which flight speeds are selected as $0.56 \mathrm{Ma}$ and $0.65 \mathrm{Ma}$, respectively. Flutter analysis is carried out at the initial $13 \mathrm{Km}$ flight altitude and $0^{0}$ attack angle. The time histories of the first three generalized displacements are shown in Figures 6 and 7, respectively.

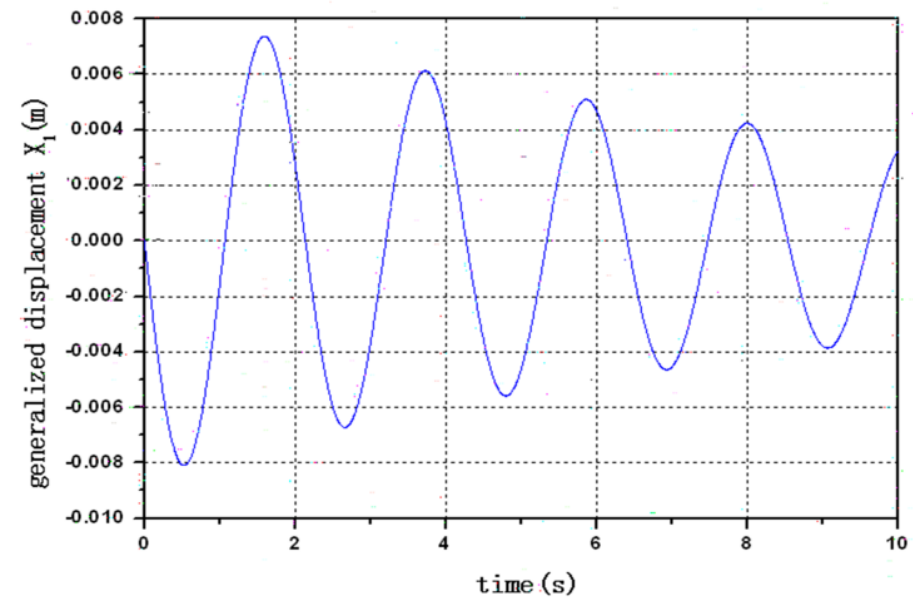

(a)

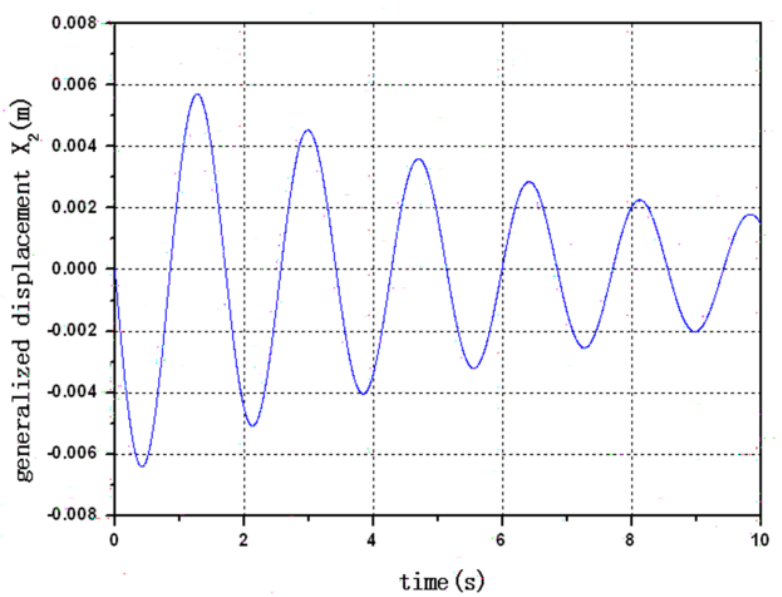

(b)

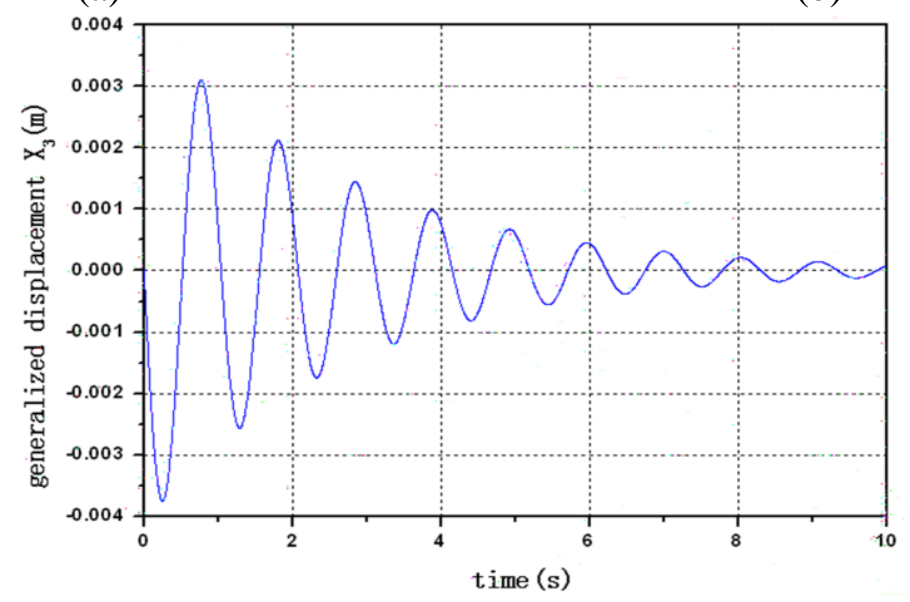

(c)

Fig.6 Time Histories of the Three Generalized Displacements with Flight Speed of 0.56 Ma (a) The First Generalized Displacement $X_{1}(\mathrm{~m})$; (b) The Second Generalized Displacement $\mathrm{X}_{2}(\mathrm{~m})$; (c) The Third Generalized Displacement $\mathrm{X}_{3}(\mathrm{~m})$ 


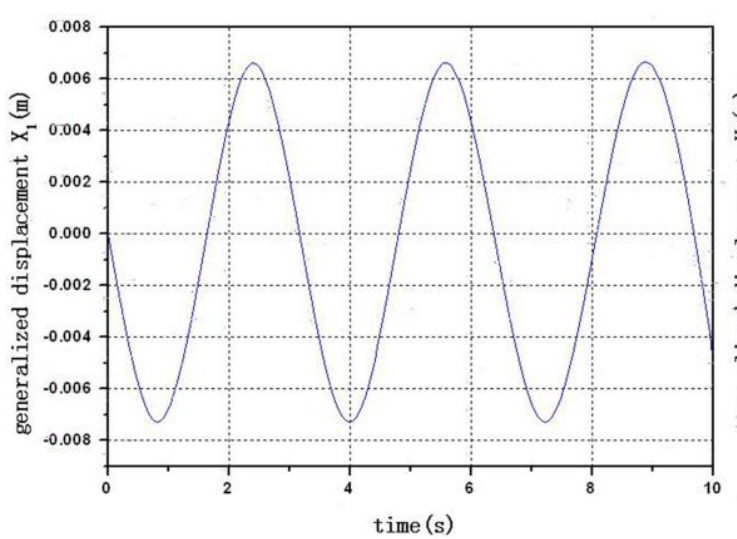

(a)

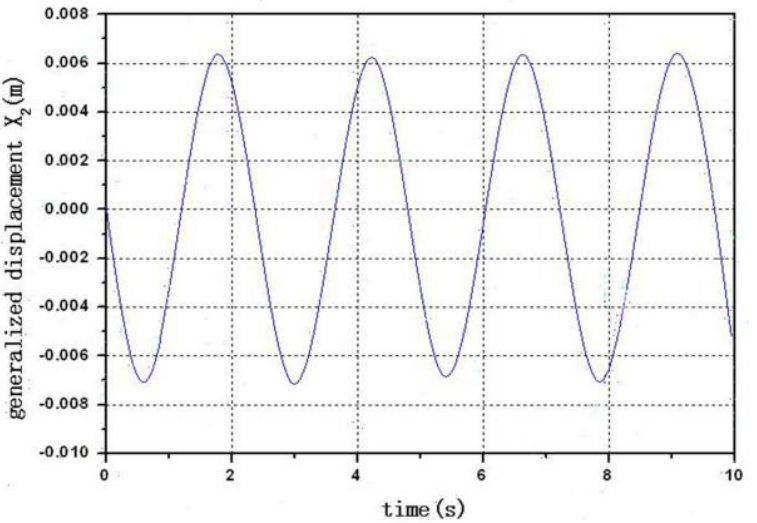

(b)

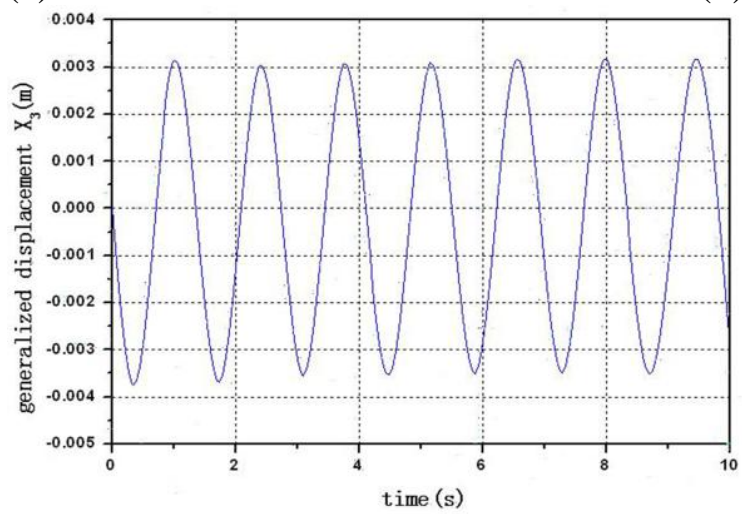

(c)

Fig.7 Time Histories of the Three Generalized Displacements with Flight Speed of 0.65 Ma (a) The First Generalized Displacement $\mathrm{X}_{1}(\mathrm{~m})$; (b) The Second Generalized Displacement $\mathrm{X}_{2}(\mathrm{~m})$; (c) The Third Generalized Displacement $\mathrm{X}_{3}(\mathrm{~m})$

The results of the study can be seen that the first three generalized displacements are convergent for the flight speed of $0.56 \mathrm{Ma}$, while the first three generalized displacements are continuous oscillations of equal amplitude for flight speed of $0.65 \mathrm{Ma}$. It shows that the flutter speed of the full composite wing is near $0.65 \mathrm{Ma}$.

\section{Conclusion}

Based on CFD/CSD coupled method, this paper has presented an effective nonlinear flutter analysis method for full composite wing with high-aspect-ratio. Some useful conclusions can be drawn as follows:

(1) Based on corotational method, a 4-node thin shell element formulation was established to analyze geometric nonlinearities. The new element is acceptable for composite thin shell structure, which is $35.3 \%$ faster computationally than the original element based on CR method.

(2) The nonlinear flutter analysis method is used to analyze to full composite wing with high-aspect-ratio, the nonlinear flutter calculation demonstrates the validity of the presented method. The flutter speed of the composite wing is near $0.65 \mathrm{Ma}$.

\section{Acknowledgment}

This study is supported by the Basic Research Foundation of Northwestern Polytechnical University (No: JC201238).

\section{References}

[1] Attar P J, Dowell E H, White J R. Modeling delta wing limit-cycle oscillations using a 
high-fidelity structural model [J]. Journal of Aircraft, 2005, 42(5): 1209-1217.

[2] Gordnier R E. Computation of limit-cycle oscillations of a delta wing [J]. Journal of Aircraft, 2003, 40(6): 1206-1208.

[3] Attar P J, Gordnier R E. Aeroelastic prediction of the limit cycle oscillations of a cropped delta wing [J]. Journal of Fluids and Structures, 2006, 22(1): 45-58.

[4] Xu M. An X M, Chen S. CFD/CSD coupling numerical computational methodology [J]. Acta Aeronautica et Astronautica Sinica, 2006, 27(1): 33-37.

[5] Gao W K. Numerical simulation of 3-D wing flutter in time domain [J]. Journal of Astronautics, 2008, 29(4): 1151-1126.

[6] Farhat C, Geuzaine P, Brown G. Applicaion of a three-field nonlinear fluid structure formulation to the prediction of the aeroelastic parameters of an F-16 fighter [J]. Comput Fluids, 2003, 32: 3-29.

[7] Crisfield M A. Non-linear finite element analysis of solids and structures. Volume 1: Essentials [J]. Wiley New York Ny, 1991.

[8] An X M, Xu M. An improved geometrically nonlinear algorithm and its application for nonlinear aeroelasticity [J]. Chinese Journal of Theoretical and Applied Mechanics, 2011, 43(1): 97-104.

[9] Baghdasaryan G Y, Mikilyan M A, Saghoyan R O, Cestino E, Frulla G, Marzocca P. Nonlinear LCO "amplitude-frequency" characteristics for plates fluttering at supersonic speeds [J]. International Journal of Non-linear Mechanics, 2015, 77: 51-60.

[10] C. Pacoste. Co-rotational flat facet triangular elements for shell instability analysis [J]. Computer Methods in Applied Mechanics and Engineering, 1998, 156: 75-110.

[11] J. M Battini. A modified corotational framework for triangular shell elements [J]. Computer Methods in Applied Mechanics and Engineering, 2007, 196: 1905-1914. 